\title{
Formação de mestres em saúde coletiva em período de pandemia por Covid-19
}

\author{
Training of masters in collective health during a pandemic period by Covid-19 \\ Formación de maestros em salud colectiva durante un período pandémico por Covid-19
}

Recebido: 27/07/2021 | Revisado: 03/08/2021 | Aceito: 06/08/2021 | Publicado: 10/08/2021

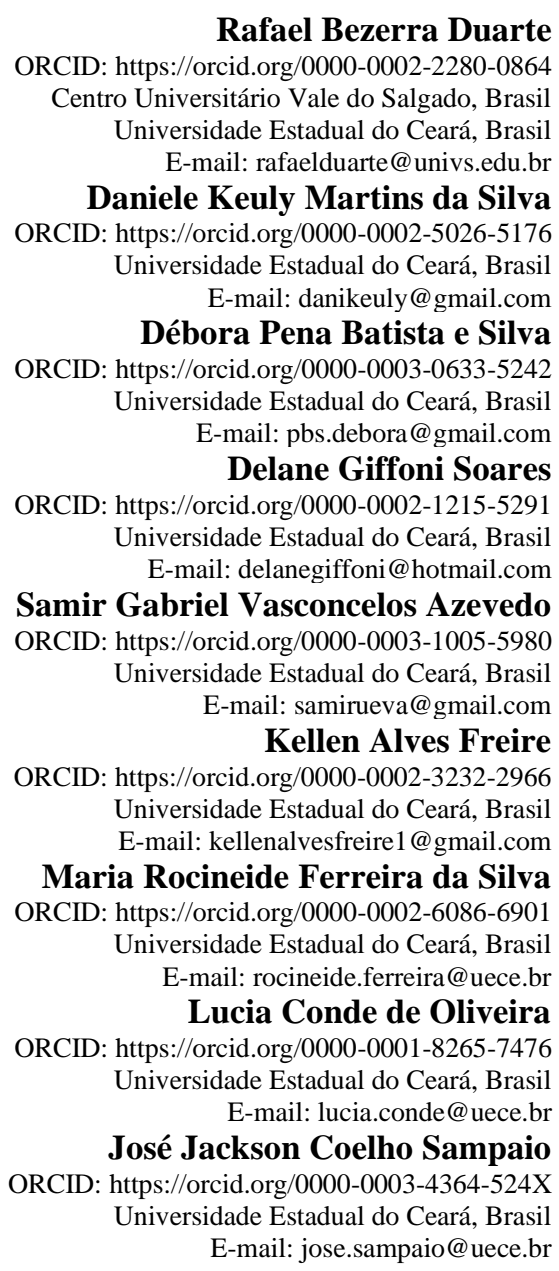

\section{Resumo}

A Saúde Coletiva é considerada um campo de investigação e produção de conhecimento, sendo compreendida como uma prática social e de natureza interdisciplinar que congrega três áreas: a Epidemiologia, as Ciências Sociais e Humanas em Saúde e a Política, Planejamento e Gestão em Saúde. Objetivou-se descrever as experiências, desafios e contribuições da disciplina de Política, Gestão e Planejamento em Saúde para a formação de mestres em Saúde Coletiva, no período atual de pandemia por Covid-19. Trata-se de um estudo descritivo, do tipo relato de experiência, realizado no período de fevereiro a junho de 2020, durante o desenvolvimento das atividades realizadas na disciplina supracitada no Programa de Pós-Graduação em Saúde Coletiva da Universidade Estadual do Ceará. A disciplina propiciou um espaço remoto de experiências, rico em novos saberes e práticas, além de oportunizar um ambiente de discussão e compartilhamento de conhecimentos, assim como conduzir os discentes a produzirem pensamentos mais críticos e reflexivos. A disciplina constituiu-se, portanto, como uma experiência enriquecedora e de grande relevância para a formação dos mestrandos em Saúde Coletiva. Além disso, possibilitou aos mestrandos a (des)construção e consolidação de conceitos, a superação de obstáculos e dificuldades.

Palavras-chave: Ensino; Infecções por Coronavírus; Políticas públicas de saúde; Programas de pós-graduação em saúde; Saúde pública. 


\begin{abstract}
Collective Health is considered a field of investigation and knowledge production, being understood as a social practice and of an interdisciplinary nature that brings together three areas: Epidemiology, Social and Human Sciences in Health and Health Policy, Planning and Management. To describe the experiences, challenges and contributions of the discipline of Health Policy, Management and Planning for the training of masters in Public Health, in the current period of pandemic by Covid-19. This is a descriptive study, of the experience report type, carried out from February to June 2020, during the development of activities carried out in the aforementioned discipline in the Graduate Program in Public Health at the State University of Ceará. The course provided a remote space for experiences rich in new knowledge and practices, in addition to providing an environment for discussion and sharing of knowledge, as well as leading students to produce more critical and reflective thoughts. The subject was, therefore, an enriching experience of great relevance for the training of Master's students in Public Health. In addition, it enabled the master's students to (de)construction and consolidation of concepts, overcoming obstacles and difficulties.
\end{abstract}

Keywords: Teaching; Coronavirus infections; Health policy; Health postgraduate programs; Public health.

\title{
Resumen
}

La Salud Colectiva es considerada un campo de investigación y producción de conocimiento, entendiéndose como una práctica social y de carácter interdisciplinario que aglutina tres áreas: Epidemiología, Ciencias Sociales y Humanas en Salud y Política de Salud, Planificación y Gestión. Para describir las experiencias, Retos y aportes de la disciplina de Política, Gestión y Planificación Sanitaria para la formación de Maestros en Salud Pública, en el actual período de pandemia por Covid-19. Se trata de un estudio descriptivo, del tipo informe de experiencia, realizado de febrero a junio de 2020, durante el desarrollo de las actividades realizadas en la referida disciplina en el Programa de Posgrado en Salud Pública de la Universidad Estatal de Ceará. El curso proporcionó un espacio remoto para experiencias ricas en nuevos conocimientos y prácticas, además de proporcionar un entorno para la discusión y el intercambio de conocimientos, además de llevar a los estudiantes a producir pensamientos más críticos y reflexivos. La asignatura supuso, por tanto, una experiencia enriquecedora y de gran relevancia para la formación de los estudiantes de Máster en Salud Pública. Además, permitió a los alumnos del máster la (des)construcción y consolidación de conceptos, superando obstáculos y dificultades.

Palabras clave: Enseñanza; Infecciones por Coronavírus; Política de salud; Programas de posgrado en salud; Salud pública.

\section{Introdução}

A Saúde Coletiva (SC) passa a existir no Brasil por volta dos anos 1970, em meio ao regime militar, sob a intensa influência de um pensamento de esquerda, democrático, não dogmático e aberto, frontalmente contrário à ditadura militar. A SC foi produzida no movimento da Reforma Sanitária (Paim, 2008; Paiva \& Teixeira, 2014).

De modo geral, a SC pode ser compreendida em seu processo de constituição histórica como um campo de produção de conhecimentos bastante complexo, tendo por base a problemática da saúde, da doença e do cuidado, os quais se desdobram em processos de desenvolvimento e experimentação tecnológica no campo das práticas sociais, de promoção, de proteção da saúde e de prevenção de riscos e agravos que envolva desde a formulação e implementação de políticas públicas de caráter intersetorial até a realização de atividades que se materializam na prática diária dos serviços de saúde e de organizações sociais voltadas para a melhoria das condições e dos modos de vida de indivíduos e grupos da população (Campos et al., 2017; Silva, Schraiber \& Mota,2019).

De acordo com Paim e Almeida Filho (1998), a SC pode ser compreendida como uma prática social, uma construção histórica que supõe o redimensionamento teórico da saúde como campo de força da produção científica. É também um espaço pelas quais diferentes instituições e organizações sociais, constituídas por vários agentes, realizam ações concretas de promoção da saúde.

Para Osmo e Schraiber (2015), a SC é um campo de investigação e produção de conhecimento, no qual deve haver um diálogo entre diversas disciplinas na constituição de um objeto complexo, a saúde em sua dimensão social. Entretanto, é também um campo de intervenção sobre esse mesmo objeto que surge de um conjunto de ideias éticas normativas da equidade e bemestar. 
Além disso, a SC pode ser considerada como um campo de conhecimento de natureza interdisciplinar, cujas áreas básicas são a Epidemiologia, as Ciências Sociais e Humanas em Saúde e a Política, Planejamento e Gestão em Saúde (PPGS). A Epidemiologia é fruto direto da saúde pública, sendo considerada uma disciplina por excelência do campo. As Ciências Sociais e Humanas em Saúde trazem contribuições importantes para a reflexão crítica na área. Contudo, os autores apontam que é na política, no planejamento e na gestão que a dimensão de intervenções se solidifica concretamente. Juntas, essas disciplinas buscam investigar a situação de saúde, a relação entre os processos de trabalho, patologias e agravos, as intervenções de grupos e classes sociais a respeito de questões sanitárias, bem como as políticas públicas (Baptista, Azevedo \& Machado, 2015).

Ainda segundo Baptista, Azevedo e Machado (2015), são nas discussões das políticas de saúde, planejamento e gestão que acontece o recrutamento das evidências epidemiológicas, as análises dos movimentos sociais, bem como a reflexão crítica das ciências sociais e humanas em saúde, para que sejam postas em ato, na produção de ações dirigidas à promoção ou recuperação da saúde da população.

A constituição e a consolidação da SC implicam e exigem a formação de profissionais capazes de se inserir em processos de produção de conhecimentos e de tecnologias, assim como na formulação e implementação de políticas e práticas, as quais assumam como objeto as condições, os estilos e os modos de vida de distintos grupos populacionais. Atuando nessa trajetória, uma conversação com outros campos disciplinares e outras áreas de prática, como por exemplo, a política, a sociologia, a economia, a clínica, a antropologia, o direito, entre outras áreas (Leal \& Freitas, 2006; Silva, Schraiber \& Mota, 2019).

Leal e Freitas (2006), ainda apontam que os programas de pós-graduação no campo da SC, sejam eles lato sensu (especialização, residência) ou stricto sensu (mestrados e doutorados), têm sido espaços privilegiados para o processo de produção de conhecimentos e experimentação tecnológica. Referenciam ainda que os cursos de mestrado e doutorado na área vêm se ampliando ao longo dos anos e consolidando um conjunto heterogêneo de grupos e linhas de pesquisa que definem, de certo modo, os limites e a configuração do campo.

Nesta perspectiva, tem-se observado que nos últimos anos o ensino das disciplinas do campo da SC, assim como o desenvolvimento de atividades pedagógicas que reproduzem as práticas características desse campo, vêm se institucionalizando em vários âmbitos e níveis do processo de formação de pessoal na área de saúde.

Nesse contexto, tendo em vista proporcionar aos mestrandos um espaço para refletirem em torno dos processos políticos e de formulação e gestão das políticas de saúde, o Programa de Pós-Graduação em Saúde Coletiva (PPSAC) da Universidade Estadual do Ceará (UECE) oferta no primeiro ano de mestrado acadêmico em SC a disciplina Política, Gestão e Planejamento em Saúde (PGPS).

Em virtude da crise sanitária instaurada pela Covid-19, medidas mais restritivas, como o isolamento social, foram estabelecidas como forma de conter a propagação do vírus. Não foi diferente com as instituições de Ensino Superior que precisaram se adaptar a uma nova realidade com o uso de uma tecnologia já conhecida, porém pouco explorada, a utilização de aulas remotas (Vercelli, 2020). Ressalta-se que as atividades da disciplina, antes iniciada presencialmente, deram continuidade de forma remota para que o ensino-aprendizagem não fosse prejudicado.

O ensino remoto, praticado atualmente, devido a pandemia da Covid-19, assemelha-se à Educação a Distância (EaD), mas isso apenas no que se refere a utilização de uma educação mediada pela tecnologia digital. Os princípios desse novo modelo de 'educação remota' acompanham os da educação presencial. Salienta-se que o termo 'remoto' se refere apenas à mudança do espaço físico que outrora era presencial e agora, provisoriamente e por algum tempo, é digital, termo muito usado na área de Tecnologia de Informação (TI) o qual se refere à não presença do aluno em sala de aula presencial (Joye, Moreira \& Rocha, 2020).

Diante desse novo cenário, surgem as seguintes perguntas norteadoras: Quais as experiências vivenciadas pelos discentes na disciplina de PGPS no programa de mestrado acadêmico em SC da UECE? Quais os desafios enfrentados na 
disciplina durante as aulas remotas no período de pandemia da Covid-19? Quais as contribuições da disciplina para a formação de mestres em SC?

Deste modo, espera-se que o presente relato de experiência venha possibilitar a ampliação de novos conhecimentos relacionados às experiências vivenciadas dentro dos programas de mestrado em SC, bem como ressaltar a importância das disciplinas para o amadurecimento e a formação de profissionais mais críticos e reflexivos na área da saúde coletiva para que estes possam oferecer contribuições para o desenvolvimento de novas pesquisas na área.

O presente artigo tem por objetivo descrever as experiências, desafios e contribuições da disciplina de PGPS para a formação de mestres em Saúde Coletiva no período de pandemia por Covid-19.

\section{Metodologia}

Trata-se de um estudo descritivo, do tipo relato de experiência. Segundo Holliday (2006), este tipo de estudo permite a sistematização da experiência de maneira a promover por meio de uma reconstrução coordenada, de análise, síntese, indução e dedução, pela interpretação crítica dos episódios vivenciados, levando em consideração o contexto histórico no qual os sujeitos estão inseridos, tendo em vista compartilharem com outras pessoas o que foi apreendido.

A experiência aconteceu no primeiro semestre de 2020, especificamente durante o período de fevereiro a junho, durante o desenvolvimento das atividades realizadas na disciplina de PGPS do PPSAC da UECE. O campus sede da Universidade fica localizado no município de Fortaleza, Ceará.

Na presente experiência, os sujeitos envolvidos foram os discentes e os professores permanentes da disciplina. À princípio, com aulas presenciais, uma vez por semana por quatro meses, totalizando 60 horas aula. O método de aula escolhido pelos professores foi uma construção coletiva, disponibilizando materiais de apoio com antecedência, sendo propostas rodas de conversa e grupos de discussão, apresentando também, seminários com imersão, no qual os grupos contextualizavam e se colocavam a partir das leituras prévias.

Entretanto, no mês de março foi declarado lockdown no Estado do Ceará, um isolamento social mais rígido, popularmente conhecido como quarentena, devido à pandemia provocada pela Covid-19. As aulas passaram a ser remotas por segurança em saúde, mas tomou-se o cuidado de preservar a didática já estabelecida para a disciplina. Houve o prazer de conhecer, discutir, ouvir e relatar experiências diante dos seguintes temas: Estado ampliado e sociedade civil em Gramsci, Reforma Sanitária como fenômeno sócio-histórico e seu contexto de transição democrática, Diretrizes para organização e financiamento do SUS, entre outros.

No que se refere aos aspectos éticos, o presente estudo não foi submetido para avaliação de um Comitê de Ética em Pesquisa, tendo em vista seu formato e metodologia adotada. Todavia, foram respeitados os princípios bioéticos adotados em pesquisas durante todo o processo de construção desse manuscrito descritivo.

\section{Resultados e Discussão}

A disciplina PGPS do PPSAC da UECE iniciou-se de forma presencial, sendo realizados somente cinco encontros nesse tipo de modalidade, prosseguindo com aulas remotas, contabilizando nessa nova conformação online mais 10 aulas, devido ao isolamento social decretado pelo governador do Estado para conter a expansão rápida da pandemia, causado por um vírus denominado novo coronavírus (SARS-CoV-2), que ocasiona a Covid-19. Lecionada por dois professores, juntos, elaboraram o plano de ensino da disciplina visando proporcionar aos discentes um espaço de discussão e reflexão mais amplo sobre os elementos teóricos e metodológicos para a análise de política de saúde, práticas, planejamento e gestão em saúde, bem como sobre os processos de organização do Sistema Único de Saúde (SUS). 
A presente disciplina tem como objetivos: Discutir o enfoque teórico do campo da SC e as abordagens metodológicas para o estudo de políticas de saúde; Analisar o desenvolvimento das políticas de saúde no Brasil, a caracterização dos processos políticos geradores das práticas de planejamento e gestão em saúde nas diferentes conjunturas do país; Descrever a trajetória política da Reforma Sanitária Brasileira; Analisar a situação atual e as perspectivas das políticas de saúde no Brasil; Desenvolver compreensão crítica sobre a organização de sistema de serviços de saúde e de suas articulações com os princípios e as diretrizes do SUS; Identificar o desenvolvimento conceitual e metodológico do planejamento em saúde na América Latina; Identificar as correntes de pensamento na área de planejamento e gestão em saúde no Brasil.

Em relação à didática proposta pelos professores, a disciplina foi trabalhada a partir de exposição dialogada, discussão sobre textos indicados, com leitura prévia de acordo com as temáticas propostas na ementa pedagógica, construção de sínteses, resenhas críticas, e apresentação de seminário pelos mestrandos. No que se refere à avaliação dos discentes na disciplina, a mesma foi feita por meio de participação nas aulas, frequência, realização e entrega de atividades e as apresentações dos seminários.

Pontuando sobre as aulas remotas, foi percebida a preocupação dos professores em buscar novos métodos e formas para a continuidade da disciplina por meio do uso das tecnologias digitais. Essa situação exigiu alterações no cronograma das aulas, entretanto, o conteúdo programático foi contemplado. As aulas aconteceram por meio do uso do aplicativo Google Meet.

Experiências semelhantes também podem ser observadas em outros estudos. Moura et al. (2020) descrevem em seu artigo que, frente a pandemia da Covid-19, as atividades pedagógicas do Programa de Residência Multiprofissional em Gestão de Políticas Públicas para a Saúde da Escola Superior de Ciências da Saúde do Distrito Federal, também tiveram que passar por processo de adaptação, fazendo-se o uso do ensino remoto síncrono emergencial. Logo, as atividades foram realizadas por meio do método da gamificação, designadas "Que a força esteja com o SUS!". Nesse novo processo de ensino, foram utilizadas as ferramentas tecnológicas digitais de apoio: Google Classroom, Google Meet e o Instagram. Nesta experiência pode-se evidenciar, a necessidade de ajustes no planejamento e cronograma de atividades, assim como, o engajamento de todos os envolvidos do programa de residência, maior autonomia e protagonismo dos residentes.

Jacinto (2021) descreve em seu relato de experiência acerca da condução da disciplina de Estágio Básico em Psicologia Social em uma instituição de ensino superior, durante o período de ensino remoto emergencial devido à pandemia da Covid-19. O autor destaca que, para a condução da referida disciplina, também se fez o uso do Ambiente Virtual de Aprendizagem adotado pela instituição, e que nesse ambiente existe espaços para postagem dos conteúdos, assim como possibilitou um local de comunicação assíncrona entre discentes e docentes, buscando garantir a troca e construção coletiva de saberes. Além disso, foi utilizada a plataforma Teams® para a efetivação das aulas síncronas. Nestas, buscou-se adotar a metodologia do estudo de caso, os quais foram conduzidos por meio de leitura de textos, leitura de documentos reais com identificação preservada, realização de questionário, apresentação de tabelas e gráficos, exibição de documentários e aula expositiva.

A pandemia da Covid-19 fez com que todos os estabelecimentos considerados não essenciais ficassem fechados, assim como a suspensão das aulas, ocasionando o isolamento social, o qual foi visto como uma das formas de prevenção e enfrentamento do problema. Todavia, no dia 17 de março de 2020, em caráter excepcional e urgente, foi lançada, pelo Ministério da Educação brasileiro, a Portaria 343, a qual trata sobre a substituição das aulas presenciais por aulas mediadas por Tecnologias da Informação e Comunicação (TIC) (Brasil, 2020).

Um ponto marcante da disciplina foi à forma como os professores conduziam as discussões em cada encontro remoto e até mesmo nos seminários. Os educadores mostraram novas possiblidades para a construção do conhecimento. Estes acompanhavam atentamente as discussões, que eram iniciadas por eles com uma apresentação inicial sobre os textos que seriam trabalhados nas aulas/seminários, posteriormente sendo a discussão conduzida pelos discentes. Na maioria das vezes, os 
professores apontavam assuntos que precisavam ser mais bem desenvolvidos, apresentavam novos esclarecimentos sobre o que estava sendo abordado e, por vezes, contextualizavam em termos históricos e políticos, ou ainda, faziam relação com o pensamento de outros autores.

Neste sentido, Freire (2003) descreve que os educadores precisam criar as possibilidades para a produção ou construção do conhecimento pelos alunos (as), em um processo em que o professor e o aluno não se reduzam à condição de objeto um do outro. Além disso, o autor reafirma que "ensinar não é transferir conhecimento, mas criar as possibilidades para sua própria produção ou a sua construção" (Freire, 2003, p. 47), assim como aponta que o conhecimento precisa ser vivenciado e testemunhado pelo agente pedagógico.

A partir da experiência vivenciada nas atividades desenvolvidas na disciplina, pode-se observar principalmente nas aulas remotas o quanto somos capazes de (des)construir e apreender novos saberes e novas práticas. Observamos também o quanto a disciplina nos exigiu de criar um maior hábito de leitura para que fosse possível buscar um maior conhecimento e assim tornarmo-nos profissionais mais críticos e reflexivos.

Cavéquia, Maciel e Rezende (2010), afirmam que os indivíduos precisam possuir habilidades e conhecimentos para assim interpretarem e analisarem de maneira crítica e autônoma o crescente número de informações e que, para isso, se faz necessário ter a rotina de leitura. Neste sentido, os estudantes devem ser capazes de transitarem pelas informações e transformálas em conhecimento por meio da reflexão e da criticidade, para que possam estar inseridos na sociedade atual.

De acordo com Villardi (1999, p.6) a "interpretação pressupõe individualidade, pressupõe a possibilidade de imprimir a própria marca naquilo que leu". Deste modo, é pela leitura crítica, que o discente apreende e aprende a manifestar-se sobre o conhecimento adquirido. Para Yunes (2011, p.31), é por meio da leitura crítica que, "o leitor comenta e replica o que lê, com juízo e tomada de posição".

Tendo em vista a emergência causada pela Covid-19, as instituições de ensino fechadas, segundo Joye, Moreira \& Rocha (2020), professores e alunos foram estimulados a dar continuidade ao processo de ensino-aprendizagem em seus lares, por meio das TIC. Contudo, os autores apontam que em algumas situações, ambos, professores e alunos enfrentaram dificuldades no início dessa nova realidade.

Diante dessa situação, um dos professores teve que lidar com as mudanças e com as surpresas da prática docente, mas relatou apresentar dificuldades com esse novo formato de aula, pois nunca tinha passado por algo parecido. Todavia, enfrentou todos os obstáculos e tornou as aulas não presenciais síncronas prazerosas, leves e, ao mesmo tempo, com ganhos consideráveis de conhecimentos. Destacamos ainda que, por essa metodologia, foram realizadas riquíssimas construções, reflexões e partilhas de saberes, bem como foi um espaço para a apresentação dos seminários, conduzidos pelos próprios discentes. O fato é que, mesmo sem os corpos fisicamente presentes, os conhecimentos foram construídos de forma positiva.

Redecker (2017) afirma que, entre os diversos desafios do ensino remoto, destaca-se o papel primordial dos professores e a responsabilidade institucional de apoiá-los. Foi necessário dar respostas às especificidades do planejamento de ensino-aprendizagem remoto, facilitar o desenvolvimento de competências digitais, contribuir com o domínio de ferramentas e recursos tecnológicos educacionais, viabilizar novas formas de avaliação, de mediação e facilitação da aprendizagem em ambiente digital. Havia familiaridade com o uso do ambiente virtual de aprendizagem, mas, não era uma política instrucional. Da mesma forma, não havia experiência com os recursos de teleconferência para uso rotineiro pelos docentes, e ela era reduzida ao uso de recursos para gravação e disponibilização de aulas incluindo vídeos. Era primordial apoiar o desenvolvimento de competências digitais pelo corpo docente.

Além disso, pode-se evidenciar, a partir dessa experiência, o quanto é necessário a disciplina na matriz curricular do programa de mestrado de SC, tendo em vista trazer grandes contribuições para a formação de mestres em saúde coletiva, uma vez que, essa disciplina propicia aos discentes um espaço para a discussão acerca dos processos históricos os quais permitiram 
o desenvolvimento dos sistemas, das políticas, do planejamento e gestão em saúde no Brasil, trazendo como destaque o movimento da Reforma Sanitária Brasileira e a construção do SUS.

Também foi possível vivenciar, na disciplina, discussões sobre as experiências inovadoras de planejamento e gestão que acontecem em instituições gestoras do SUS ao nível, estadual e municipal, assim como se pode debater sobre as normas instituídas pelo Ministério da Saúde (MS), as quais vêm definindo a organização dos sistemas e serviços de saúde. A disciplina proporcionou aos mestrandos uma discussão ampliada a respeito da articulação entre atenção e gestão na organização das redes de atenção à saúde, na perspectiva da universalidade, da integralidade, da equidade, da humanização e com a participação da comunidade. Logo, destacamos que todas essas discussões proporcionadas pela disciplina nos deram condições para a construção de um conhecimento mais reflexivo e crítico acerca dos elementos teóricos e metodológicos das políticas de saúde, gestão e planejamento em saúde, bem como sobre os processos de organização do SUS.

Na disciplina, os mestrandos passaram a entender de fato o conceito de política de saúde, o qual é apresentado por Paim e Teixeira (2006) como sendo uma resposta social de uma organização perante as condições de saúde dos indivíduos e das populações e seus determinantes. Os autores referem-se ainda que a política de saúde é como uma relação entre a produção, distribuição, gestão e regulação de bens e serviços que afetam a saúde das pessoas e do ambiente. Enquanto discentes, passamos então a compreender que a política de saúde abrange questões referentes ao poder em saúde, assim como as que se aludem ao estabelecimento de diretrizes, planos e programas de saúde.

Assim, enquanto disciplina dentro do mestrado, pode-se observar que quando estudados os assuntos sobre política de saúde, os mesmos buscam compreender as relações de poder na conformação da agenda, na formulação, na condução, na implementação e na avaliação de políticas. Destacamos que apreender essas relações são essenciais no ensino da saúde coletiva. Outra contribuição importante obtida na disciplina foi o entendimento e compreensão que tivemos a respeito das relações entre a política de saúde com políticas econômicas e sociais, controle social, economia da saúde e financiamento.

No que se refere ao planejamento em saúde, a disciplina nos forneceu conhecimentos relacionados às estratégias que podem ser utilizadas para o enfrentamento de problemas, sobre os principais mecanismos que são utilizados para implementação de uma política, assim como os métodos que são usados para construção de planos, programas e projetos de saúde. Na parte que envolve a gestão em saúde, as contribuições obtidas na disciplina foram essenciais para os futuros mestres, uma vez que no campo da saúde coletiva precisamos entender sobre a criação e utilização de meios que possibilitem concretizar os princípios de organização de políticas, gestão de serviços e sistemas de saúde e o seu financiamento. Ressaltamos que muitos desses conhecimentos foram obtidos pelas leituras recomendadas pelos professores.

Ainda, ficou evidenciado que a disciplina representa um espaço importante para os discentes dentro dos programas de mestrado em saúde coletiva, tendo em vista que essa disciplina tem por finalidade mobilizar mestrandos a refletirem em torno dos modos de produzir conhecimento e suas finalidades. Baptista, Azevedo e Machado (2015), na obra Políticas, Planejamento e Gestão em Saúde: abordagens e métodos de pesquisa, trazem para os leitores justamente esse espaço de reflexão no que se refere aos modos de produzir os conhecimentos e suas finalidades.

Não podemos esquecer que a experiência vivenciada na disciplina ainda nos possibilitou apreender como os professores utilizam as estratégias/métodos de ensino, refletirmos sobre a prática docente, além de contribuir para o aperfeiçoamento desta prática, e reconhecermos que o processo de ensino-aprendizagem é um desafio na vida do professor. As vivências em todos os processos metodológicos experimentados na disciplina constituíram-se, portanto, de uma experiência enriquecedora e de grande relevância para a formação de futuros profissionais docentes na área da saúde coletiva, possibilitando deste modo, a construção e consolidação de conceitos, a quebra de barreiras e a superação de dificuldades da carreira de docente no ensino superior. 
Diante desse contexto, Tardiff (2009), descreve que é no ambiente do trabalho, por meio da experiência diária do docente na sala de aula que se desenvolve a aprendizagem da docência e essa, com reflexões sistemáticas e com o auxílio da teoria, busca aproximar os alunos da realidade das práticas de ensino-aprendizagem, bem como da carreira docente.

Como já foi descrito, no decorrer da disciplina aconteceu situação inesperada com a paralização das aulas por conta da pandemia causado pela Covid-19, o que acarretou em alteração do cronograma, assim como a busca por novas estratégias, táticas e métodos de ensino-aprendizagem, mostrando assim que os professores precisam apreender a lidar com as mudanças e com as surpresas da prática docente.

Por termos dois professores lecionando na disciplina, tivemos a oportunidade de ter o contato com diferentes visões do ensino, como também abordagens distintas de prática e da didática dentro do ensino superior. Este contato possibilitou o compartilhamento de experiências adquiridas por cada um dos professores, complementando e fortalecendo a formação de futuros mestres.

A atividade docente é bastante complexa e abrange não apenas os saberes técnicos específicos, envolve também os saberes afetivos, sociais, psicológicos, políticos, éticos e principalmente os saberes pedagógicos. Logo, estes saberes são influenciados pelos contextos de formação e construção dos docentes e pelas histórias de vida de quem ensina e de quem aprende. Todavia, é durante o processo de docência que se constroem valores, se formam relações sociais e ocorrem à criação de vínculos, esses que irão definir a formação de profissionais e de sujeitos (Soares \& Cunha, 2010).

Portanto, a experiência relatada foi marcada por grandes descobertas, em que os discentes compreenderam a importância dos enfoques dados ao componente curricular no âmbito da saúde coletiva. É perceptível a importância do desenvolvimento de atividades que contribua tanto no ensino-aprendizagem dos discentes, como também na troca de conhecimentos com o docente na perspectiva de troca mútua entre aluno-professor para que seja possível fomentar sua prática.

\section{Conclusão}

A experiência vivenciada na disciplina de PGPS do PPSAC da UECE compartilhada neste trabalho possibilitou conhecer estratégias de ensino, compartilhar o conhecimento entre mestrandos e professores, refletir sobre a prática de ensinoaprendizagem em tempos de pandemia, além de contribuir para o aperfeiçoamento desta e reconhecer que o processo é contínuo, uma constante na vida dos envolvidos.

A partir dos resultados apresentados podemos observar que a disciplina, além de propiciar um ambiente de experiências para os mestrandos construir e apreender novos saberes e práticas foi proporcionado um espaço para discussão e reflexão a respeito dos elementos teóricos e metodológicos para a análise de políticas, práticas, planejamento e gestão em saúde, bem como sobre os processos de organização do SUS.

A disciplina estimula aos mestrandos uma forma de refletir mais criticamente, capacidade de se expressar de forma escrita e oral com base nas literaturas. Ainda foi possível discutir e debater as temáticas com espírito de agregar novos conhecimentos que, certamente, auxiliarão na vida dos futuros mestres.

Ressalta-se também, a importância dos conhecimentos adquiridos acerca dos conteúdos do componente curricular, ligados às políticas de saúde, planejamento e gestão em saúde para a formação de sanitaristas com habilidades e competências relacionadas ao trabalho em saúde coletiva.

As atividades vivenciadas na disciplina constituem-se, portanto, como uma experiência enriquecedora e de grande relevância para a formação dos futuros mestres em saúde coletiva, possibilitando a (Des)construção e consolidação de conceitos, a quebra de barreiras e a superação de dificuldades que poderão ser enfrentadas na carreira profissional na área da saúde coletiva, além de colaborar diretamente com a formação de futuros docentes. 


\section{Agradecimentos}

Ao Programa de Pós-graduação em Saúde Coletiva (PPSAC) da Universidade Estadual do Ceará (UECE). E ao Conselho Nacional de Desenvolvimento Científico e Tecnológico (CAPES), bolsa de estudo do mestrado.

\section{Referências}

Baptista, T. W., Azevedo, C. S., \& Machado, C. V. (2015). Políticas, Planejamento e Gestão em Saúde: abordagens e métodos de pesquisa. Rio de Janeiro: editora Fiocruz.

Brasil. Ministério da Educação (2020). Portaria 343, de 17 de março de 2020. Dispõe sobre a substituição das aulas presenciais por aulas em meios digitais enquanto durar a situação de pandemia do Novo Coronavírus-Covid-19.

Campos, G. W. S., et al. (2017). Tratado de Saúde Coletiva. Revista \& Aumentada. (2a ed.). Editora: Hucitec.

Cavequia, M. A. P., Maciel, A. G., \& Rezende, L. A. (2010). Formação do leitor: criticidade e autonomia. Revista Contrapontos. 10(3): 299-306.

Freire, P. (2003). Pedagogia da autonomia: saberes necessários à prática educativa. Paz e Terra.

Holliday, O. J. (2006). Para sistematizar experiências. Resende. (2a ed.). MMA.

Jacinto, P. M. S. (2021). Relato de experiência sobre estágio básico em psicologia social no modelo de ensino remoto emergencial. Revista Cenas Educacionais, Caetité-Bahia-Brasil, 4(10167), 1-16.

Joye, C. R., Moreira, M. M., \& Rocha, S. S. D. (2020) Educação a Distância ou Atividade Educacional Remota Emergencial: em busca do elo perdido da educação escolar em tempos de COVID-19. Research, Society and Development. 9(7), 1-27.

Leal, M. C., \& Freitas, C. M. (2006). Cenários possíveis: experiências e desafios do mestrado profissional na saúde coletiva. Editora Fiocruz.

Moura, R. A., et al. (2020). Estratégias educacionais remotas em um programa de residência multiprofissional em meio à pandemia pelo coronavírus: um relato de experiência. Revista Cenas Educacionais, Caetité -Bahia -Brasil, 3(9114), 1-19.

Osmo, A., \& Schraiber, L. B (2015). O campo da Saúde Coletiva no Brasil: definições e debates em sua constituição. Saúde Soc. 24 (supl.1), $205-218$.

Paim, J. S., \& Almeida Filho, N. (1998). Saúde coletiva: uma "nova” saúde pública ou campo aberto a novos paradigmas? Revista de Saúde Pública. São Paulo. 32(4), 299-316.

Paim, J. S., \& Teixeira, C. F. (2006). Políticas, Planejamento e gestão em saúde: balanço do estado da arte. Rev. Saúde Pública. 40 (Esp): $73-78$.

Paim, JS (2008). Reforma Sanitária Brasileira: contribuição para a compreensão e crítica. Salvador: Edufba, Fiocruz.

Paiva, C. H. A., \& Teixeira, L. A. (2014). Reforma sanitária e a criação do Sistema Único de Saúde: notas sobre contextos e autores. História, Ciências, SaúdeManguinhos [online]. 21(1), 15-36.

Redecker, C. (2017). European framework for thedigitalcompetence o Educators Dig Comp Edu. Punie Y (Ed.). Publications Office of the European Union: Luxembourg.

Silva, M. J. S., Schraiber, L. B., \& Mota, A. (2019). O conceito de saúde na Saúde Coletiva: contribuições a partir da crítica social e histórica da produção científica. Physis: Revista de Saúde Coletiva, Rio de Janeiro, 29(1), 1-19.

Soares, S. R., \& Cunha, M. I. (2010). A docência universitária e a formação para seu exercício. In: Soares, S. R., Cunha, M. I. Formação do professor: a docência universitária em busca de legitimidade. EDUFBA.

Tardiff, J. (2009). Se o professorado universitário fosse uma profissão. In: Cunha, M. I., Soares, S. R., Ribeiro, M. L. Docência universitária: profissionalização e práticas educativas. Feira de Santana: editora UEFS.

Vercelli, L. C. A. (2020). Aulas remotas em tempo de COVID-19: a percepção de discentes de um Programa de Mestrado Profissional em Educação. Revista @ mbienteeducação. São Paulo: Universidade Cidade de São Paulo. 13(2), 47-60.

Villardi, R. (1999). Ensinando a gostar de ler e formando leitores para a vida inteira. Qualitymark/Dunya.

Yunes, E. (2011). Era uma vez... a leitura. In: Yunes E. Leitores a caminho: formando agentes de leitura. Editora PUC-Rio. 